\title{
THE QUANTIFICATION AND MONITORING OF DATA QUALITY AND THE DESIGN OF OPTIMUM SWEEPS
}

\author{
Jan Brouwer $^{1}$, Peter Bakker ${ }^{1}$, Dirk Kraaijpoel ${ }^{2}$, and Kabir Roy-Chowdhury 2 \\ 1 OYO-CAG, Archimedesbaan 16 Nieuwegein, The Netherlands \\ 2 Utrecht University, Budapestlaan 4, Utrecht, The Netherlands
}

\section{INTRODUCTION}

The application of vibratory sources in seismic exploration principally allows us to tune the source signature to the (changes in) specific (sub-) surface conditions through a proper choice of the pilot signal. Objective design of source signatures is feasible only if we can define how the quality of the dataset is affected by the choice of pilot signal. This requires a description of the quality of the seismic data in terms of the pilot signal.

The quality of a seismic dataset is often determined in a rather subjective manner and is related to the interpreters appreciation of the various seismic events and their distribution in time and space. Design of pilot signals, as well as monitoring of data quality during acquisition and processing, however, require a quantitative description of the quality of the dataset.

The objective of this paper is to define a set of criteria that quantitatively describes the quality of a seismic record and to use these criteria for the monitoring of data quality during acquisition and processing and for the design of optimum sweeps.

\section{DESCRIPTIVE PARAMETERS}

In order to formulate descriptive criteria we define the seismic record in terms of the distribution in time and space of reflection signal $W(x, t)$, source coherent noise $C_{j}(x, t)$ (the subscript denotes the various types of coherent noise), and ambient noise $N(x, t)$.

$$
R(x, t)=W(x, t)+\sum_{j} C_{j}(x, t)+N(x, t)
$$

Both $W(x, t)$ and $C_{j}(x, t)$ depend on the source wavelet $S(x, t)$ whereas the ambient noise is characterized by a spatial and temporal distribution independent of the generated source signal. The quality of a dataset in a selected time-offset window is determined by:

- the distortion of the reflected signal due to ambient noise (as related to penetration);

- the distortion of the reflected signal due to source-coherent noise (as related to separation);

- the interference of the reflected signal with other reflections (as related to resolution).

We may now define the total quality of the dataset $Q_{t}$ as the weighted sum of the quality of penetration, $q_{p, i}$, separation, $q_{s, i}$, and resolution, $q_{r, i}$, in each of the windows under consideration as 


$$
Q_{t}=\frac{\sum_{i} a_{p, i} q_{p, i}+a_{r, i} q_{r, i}+a_{s, i} q_{s, i}}{\sum_{i} a_{p, i}+a_{r, i}+a_{s, i}}
$$

where $a_{x, i}$ denote the respective weights of penetration, separation, and resolution in window $i$ as subject to the interpreters preferences

The objective of Field- and Processing QC is to quantitatively monitor the degradation of the data quality in a selected set of time-offset windows and take the appropriate action should this degradation exceed a pre-defined threshold value. The objective of Sweep Design is to reduce the degradation of data quality in a set of user defined time-offset windows to a minimum through selection of an "optimum" pilot signal.

\section{QUANTIFICATION OF DATA QUALITY}

There are many ways to quantify penetration, separation, and resolution. We have chosen to adhere to a description in terms of average signal-to-noise ratio (ambient and coherent) and average duration of the source wavelet. We find respectively

$$
S N_{i}^{2}=\frac{\iint_{O_{i}} W^{2}(x, t) d x d t}{\iint_{O_{i}} N^{2}(x, t) d x d t}, S C_{i}^{2}=\frac{\iint W_{i}^{2}(x, t) d x d t}{\iint_{O_{i}} C^{2}(x, t) d x d t}, \quad L T_{i}^{2}=\frac{\iint S_{O_{i}} S^{2}(x, t) t^{2} d x d t}{\iiint_{O_{i}} S^{2}(x, t) d x d t}(\mathbf{3 a}, \mathbf{3 b}, \mathbf{3 c})
$$

as the mean-square signal-to-noise ratio, the mean-square signal-to-coherent-noise ratio, and the second order moment (Berkhout, 1984) within a time-offset window $O_{i}$. In (3c) we assume a zero phase wavelet. Normalized quality estimators are now found as

$$
q_{p, i}=\frac{S N_{i}}{S N_{0}}, \quad q_{s, i}=\frac{S C_{i}}{S C_{0}}, \quad q_{r, i}=\frac{L T_{0}}{L T_{i}}, \quad(4 \mathrm{a}, 4 \mathrm{~b}, 4 \mathrm{c})
$$

where $S N_{0}, S C_{0}$, and $L T_{0}$ respectively represent nominal signal-to-noise ratio (ambient and coherent) and wavelet duration. The total quality of the record is determined using (2). Whereas $S(x, t)$ is usually not known, its spectral representation might be deduced from the signal spectrum. Using Parseval's theorem, equations (3a-c) and (4a-c) are easily expressed in the frequency domain and $S N, S C$, and $L T$ can be determined from the signal and (coherent) noise spectra.

\section{DETERMINATION OF DATA QUALITY}

The evaluation of the data quality requires independent determination of signal, noise, and coherent noise spectra in the various time-offset windows of interest. We suggest the following approach:

1) determine $N(x, t)$ from separate noise shots; extrapolate to all other windows assuming stationary noise;

2) determine $W(x, t)$ from a window relatively undisturbed by noise; extrapolate signal to all other windows taking into account spreading $(\mathrm{dB} / \mathrm{s})$ and attenuation $(\mathrm{dB} / \mathrm{Hz})$; 
3) determine $C_{j}(x, t)$ from windows showing predominantly coherent noise (e.g., airblast, surface waves, refraction multiples);

4) transform signal and (coherent) noise to the frequency domain and determine $q_{p, i}$, $q_{s, i}, q_{r, i}$, and $Q_{t}$.

\section{DESIGN OF OPTIMUM SWEEPS}

Assuming a convolutional model both signal and coherent noise can be expressed in terms of the source signature. If we parameterize the new source signature as the perturbed version of the original linear source signature

$$
S^{*}(\omega)=a(\omega) \cdot S(\omega)
$$

we can determine the expected change in data quality from the frequency domain expression of equations (4a-c). Constraining the duration of the sweep to that of the original linear sweep ( $\int a(\omega)^{2} d \omega=1$ ), the optimal source signature may be derived through maximizing $Q_{t}$.

\section{FIELD EXAMPLE}

A field example is presented that is based on a simple test carried out at a site close to Utrecht (the Netherlands). A horizontal vibrator was used as the seismic source.

Figure 1 (top) shows the spectra of reflection signal and ambient noise for a linear sweep from 40-400 Hz. Based on these spectra, sweeps were designed that optimize penetration and resolution. The pilot signals of these two sweeps are also displayed in figure 1 (bottom).
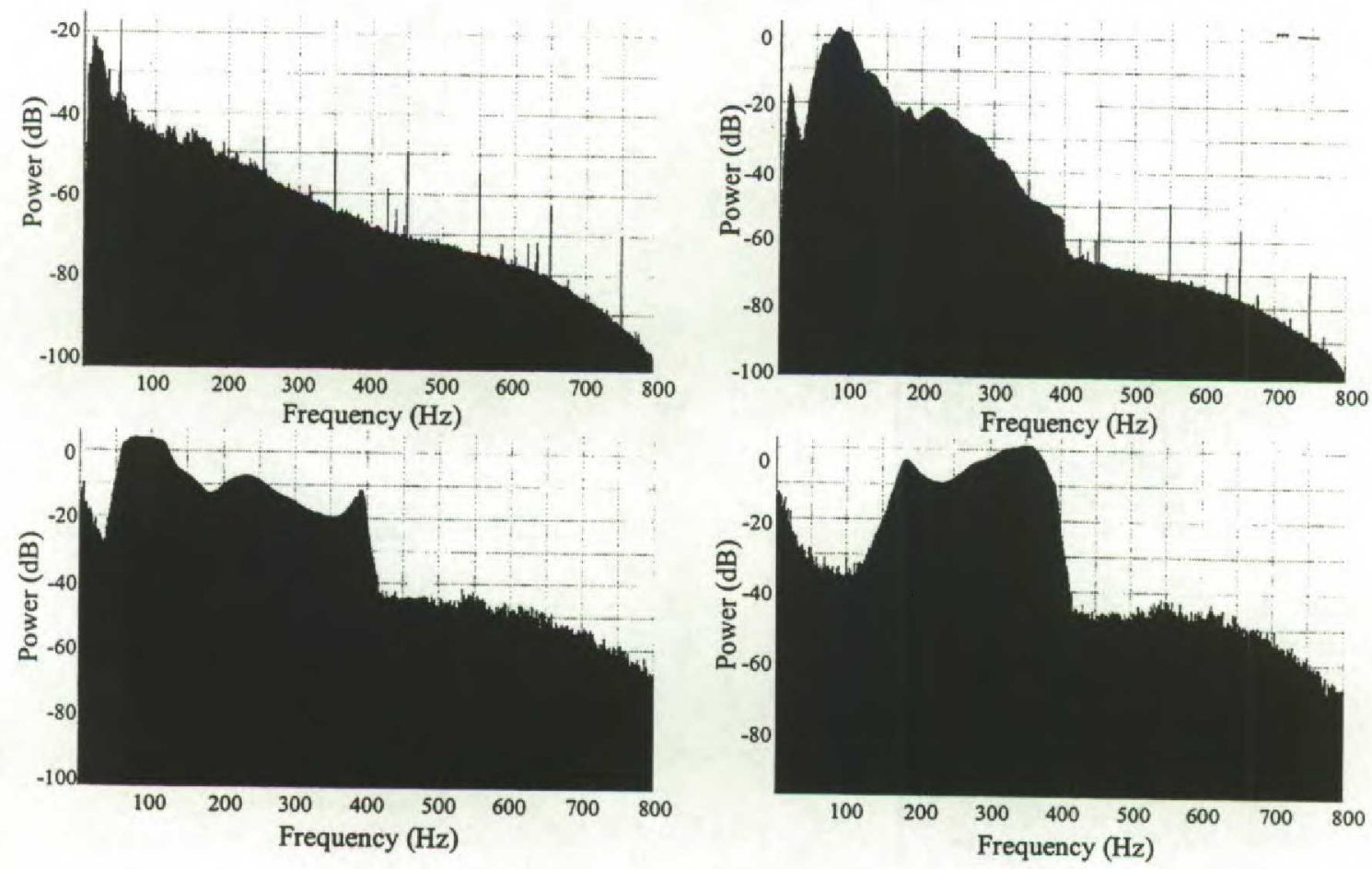

Figure 1. top: average spectra of noise (left) and reflection signal (right); bottom: pilot sweep used for optimized penetration (left) and resolution (right).

It is observed that the sweep that optimizes penetration generates most of the signal at lower frequencies (which show a high signal-to-noise ratio), whereas the sweep that optimizes resolution focuses at relatively higher frequencies (showing a low signal-to-noise ratio). 
Analysis of the data obtained for the three different sweeps reveals that the rms amplitude has changed from 790 for the linear sweep to 1470 and 127 for the sweeps that respectively optimize penetration and resolution. Dominant frequencies were found at $86 \mathrm{~Hz}, 82 \mathrm{~Hz}$, and $120 \mathrm{~Hz}$ for linear, optimized penetration, and optimized resolution sweeps.

The linear sweep as well as the sweep optimized for penetration were subsequently used to acquire the reflection profiles displayed in figure 2. It is clear from the comparison of the two identically processed and displayed profiles that the signal amplitude after optimization has considerably increased, whereas a decrease in resolution is hardly visible.
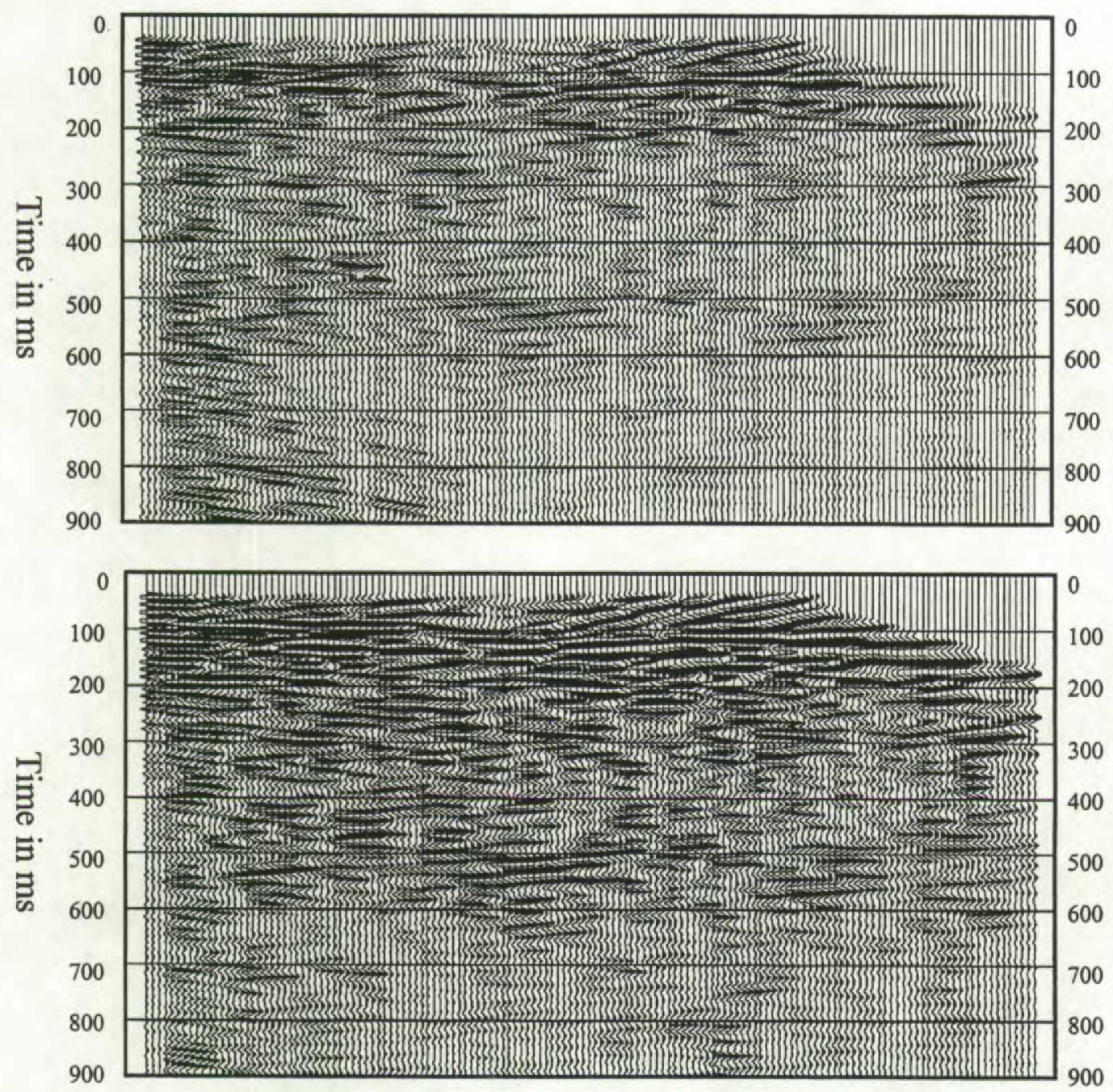

Figure 2. top: 12-fold stack reflection profile obtained with a linear sweep $(40-400 \mathrm{~Hz})$; bottom: the same reflection profile obtained with a sweep optimized for penetration.

\section{CONCLUSIONS}

It is concluded that a quantification of data quality in terms of resolution, penetration, and separation enables monitoring of the quality of the data throughout a seismic survey and allows objective design of source signals.

\section{REFERENCES}

Berkhout, A.J., 1984, Seismic resolution, resolving power of acoustical echo techniques. Handbook of Geophysical Exploration, Section I. Seismic Exploration, Geophysical Press. 